\title{
Silencing miR-199a-3p, Which Targets Integrin B8 Prevents Steroid-induced Avascular Necrosis of the Femoral Head by Regulating Osteogenesis and Adipogenesis
}

\section{Wu Yang}

The First Affiliated Hospital of Chongqing Medical University Department of Orthopaedics

\section{Yunfei Yang}

The First Affiliated Hospital of Chongqing Medical University Department of Geriatrics

\section{Minkang Guo}

The First Affiliated Hospital of Chongqing Medical University Department fo Orthopaedics

\section{Weiwen Zhu}

Sun Yat-sen University First Affiliated Hospital Department of Orthopaedics

\section{Husun Qian}

Chongqing Medical University

\section{Chengjie Lian}

The First Affiliated Hospital of Chongqing Medical University Department of Orthopaedics

\section{Haobo Bai}

The First Affiliated Hospital of Chongqing Medical University Department of Orthopaedics

\section{Hongwei Wang}

The First Affiliated Hospital of Chongqing Medical University Department of Orthopaedics

\section{Tingmei Chen}

Chongqing Medical University

\section{jian zhang ( $\square$ zhangjian@hospital.cqmu.edu.cn )}

The First Affiliated Hospital of Chongqing Medical University Department of Orthopaedics https://orcid.org/0000-0003-2908-1935

\section{Research Article}

Keywords: osteonecrosis, osteogenesis, adipogenesis, integrin beta8, miR-199a-3p, bone marrow mesenchymal stem cells, MC3T3-E1 cells

Posted Date: July 19th, 2021

DOl: https://doi.org/10.21203/rs.3.rs-709174/v1 
License: (c) (i) This work is licensed under a Creative Commons Attribution 4.0 International License. Read Full License 


\section{Abstract}

\section{Background}

The main pathogenesis of steroid-induced avascular necrosis of the femoral head (SANFH) is closely connected with osteogenesis and adipogenesis. MicroRNAs have been proved to play prominent roles in the initiation and progression of SANFH. The present study investigated the effects of silencing miR$199 a-3 p$ on the prevention and early treatment of SANFH.

\section{Methods}

RT-PCR was used to detect the expression of miR-199a-3p. Western blotting was used to detect protein expression. Alkaline phosphatase (ALP) activity, alizarin red S staining, and oil red $\mathrm{O}$ staining were used to study the effect of miR-199a-3p on the osteogenic and adipogenic differentiation of rat bone marrowderived mesenchymal stem cells (rBMSCs) and MC3T3-E1 cells. A dual-luciferase reporter assay was used to confirm the target relation of miR-199a-3p and integrin $\beta 8$ (ITGB8). Animal study was used to explore the effect of silencing miR-199a-3p in vivo.

Results

MiR-199a-3p expression was upregulated in necrotic bone tissues and rBMSCs and MC3T3-E1 cells treated with $20 \mathrm{UM}$ dexamethasone. Western blotting and a dual luciferase assay were employed to verify that miR-199a-3p directly targets ITGB8. Silencing of miR-199a-3p also promoted osteogenesis and inhibited adipogenesis of rBMSCs and MC3T3-E1 cells in the presence or absence of glucocorticoids. In addition, the silence of miR-199A-3p prevent SANFH by promoting osteogenesis in rats.

Conclusion

The upregulation of miR-199a-3p induced by GCs could reciprocally regulate the adipo-osteogenic differentiation of rBMSCs and MC3T3-E1 cells by targeting ITGB8. The miR-199a-3p-ITGB8-FAK-Erk1/2RUNX2 axis is a potential signaling mechanism, and inhibition of miR-199a-3p expression could promote osteogenesis and inhibit adipogenesis to alleviate the occurrence of SANFH.

\section{Introduction}

Steroid-induced avascular necrosis of the femoral head (SANFH), a prevalent, devasting, and refractory orthopedic disease induced by the systemic application of glucocorticoids1-3. The disease is characterized by femoral head collapse followed by substantial degeneration of the hip joint, severely restraining the life quality of patients 4 . Lack of efficient early diagnosis and treatment, the disease inescapably leads to a collapse of the femoral head followed by osteoarthritis of the hip joint5. Therefore, the patients are necessitated to undergo a hip arthroplasty6, 7. These conditions cause a heavy burden on society and families. However, to date, the detailed pathological mechanism of SANFH is still unclear. 
So far, the pathogenesis of SANFH was closely connected with the imbalance between osteogenesis and adipogenesis of bone marrow-derived mesenchymal stem cells (BMSCs)8. BMSCs are multipotential cells and considered as the progenitor of osteoblasts in the femoral head, playing an important role in the growth, regeneration, and repairment of bone tissues9. It was reported that BMSCs pools in the femoral head are impaired, the osteogenic ability of BMSCs is reduced, and the osteoblasts are abnormal in SANFH patients10. Therefore, the enhancement of osteogenesis may contribute to the prevention and early therapy of SANFH.

MicroRNAs (miRNAs) are a type of small non-coding RNAs that could bind to the 3 ' untranslated region (3'UTR) of target genes and ultimately cleaving the mRNAs or repressing translation of the target mRNAs11, 12. Recently, plenty of researches had revealed that several microRNAs were involved in the pathogenesis mechanisms of SANFH, most of which were associated with osteogenesis or adipogenesis13-16. Interestingly, recent studies have revealed that miR-199a-3p was closely associated with some orthopedic diseases including osteoporosis17, osteoarthritis18, and rheumatoid arthritis19. However, no researcher performed specific experiments to explore if miR-199a-3p is involved in the pathophysiological process of SANFH and its underlying mechanism.

In the present study, the expression of miR-199a-3p was measured. Then, the effects of miR-199a-3p on osteogenesis of rBMSCs and MC3T3-E1 cells were investigated. Furthermore, the interactions between miR-199a-3p and integrin $\beta 8$ (ITGB8) were verified. Finally, the preventative effects of miR-199a-3psilencing were examined in vitro and in vivo. These findings provide clues for the prevention and treatment of SANFH.

\section{Materials And Methods}

\section{Patients and bone tissues}

All experiments were conducted according to the Declaration of Helsinki. Every experiment was approved by the Research Ethics Committee of the Affiliated Hospital of Chongqing Medical University. Each donor signed an informed consensus with the approval of the Research Ethics Committee of the Affiliated Hospital of Chongqing Medical University. Finally, 11 SANFH (stage III and IV) patients and 11 femoral neck fracture (FNF) patients who underwent a hip arthroplasty in the First Affiliated Hospital of Chongqing Medical University were recruited. The SANFH patients and FNF patients were diagnosed by Xray and computerized tomography (CT) and further confirmed by histological analysis. All of these femoral head samples were collected after resected from the femur and were immediately divided into two halves using a bone knife. A part of the femoral head was rapidly stored in the liquid nitrogen for the next experiments, while the other part of each sample was fixed in $4 \%$ paraformaldehyde for the histological study.

\section{RNA extraction}


The bone tissues were crunched using a rongeur and ground into powders with liquid nitrogen. Following that, the miRNAs of bone tissues were extracted using a Biospin miRNA Extraction kit (BioFlux, China). Finally, Biodrop Ulite (UK) was used to detect the miRNA concentration.

\section{Real-time PCR}

The cDNA of the miRNA was synthesized using miRNA First Strand cDNA Synthesis (Sangon Biotech). A $20 \mu \mathrm{L}$ system was used for the real-time quantitative PCR reaction, and 4 secondary pores were set. The cDNA gene amplification conditions of miRNA reverse transcription were pre-denaturation $95^{\circ} \mathrm{C}$ for $10 \mathrm{~min}$, denaturation $95^{\circ} \mathrm{C}$ for $10 \mathrm{~s}$, annealing $58^{\circ} \mathrm{C}$ for $20 \mathrm{~s}$, extension $72{ }^{\circ} \mathrm{C}$ for $10 \mathrm{~s}$, and 40 cycles. The relative expression of miRNA was standardized by $U 6$ and analyzed using the $2 \Delta \Delta C T$ method. The primers were shown in Table 1.

\begin{tabular}{|ll|}
\hline \multicolumn{2}{|l|}{ Table1 Primer sequence } \\
gene name & Primer sequence \\
miR-199a-3p & F GGAACCCGTAGATCCGAACTTGTG \\
& R B661601 (Sangon Biotech) \\
U6 & F B661602 (Sangon Biotech) \\
& R B661601 (Sangon Biotech) \\
\hline
\end{tabular}

\section{Cells culture and transfection}

The rat bone marrow-derived mesenchymal stem cells (rBMSCs) and were purchased in Otwo Biotech (Guangdong, China). The human embryonic kidney (HEK)-293T cells and MC3T3-E1 cells were purchased from the National Collection of Authenticated Cell Cultures (Shanghai, China). The rBMSCs, HEK-293T cells, and MC3T3-E1 cells were respectively cultured in H/DMEM medium ( HyClone, UK) supplied with $10 \%$ fetal bovine serum (Gibco, UK), $100 \mathrm{U} / \mathrm{mL}$ penicillin, and $100 \mu \mathrm{g} / \mathrm{mL}$ streptomycin (NCM, China) at $37^{\circ} \mathrm{C}$ and $5 \% \mathrm{CO} 2$. The medium was changed every 3 days. The treat concentration of dexamethasone was $20 \mu \mathrm{M}$.

The negative control (NC), agomiR-199a-3p, antagomiR-199a-3p (antagonist of miR-199a-3p) , silTGB8, wild type-ITGB8 (WT-ITGB8) and mutant type-ITGB8 (MT-ITGB8) plasmids (GenePharma, Shanghai, China) were transfected into cells using EndofectinTM-MAX kit\GeneCopoeia, USA囚at the cell confluence of $50 \%$ in accordance with manufacturer's guidelines. The working concentration of agomiR-199a-3p was100 nM, and those of antagomiR-199a-3p, NC and silTGB8 were $200 \mathrm{nM}$.

\section{Alkaline phosphatase (ALP) activity assay}

The rBMSCs and MC3T3-E1 cells were seeded into 6-well plates and transfected. Based on the previously reported methods, the ALP activity was determined using an ALP activity detective kit (Wanlei 
Bio, China) according to the protocol of the manufacturer using a microplate reader 20, 21.

\section{Osteogenic differentiation and evaluation}

The rBMSCs and MC3T3-E1 cells were seeded into 24-well plates and transfected. When the confluence point of cells reached $80 \%$, the medium was changed as the osteogenic differentiation medium (ODM, Cyagen, USA). Alizarin red S (ARS) staining was used to evaluate the level of osteogenic differentiation. After cultured in osteogenic differentiation for 3 weeks, the rBMSCs were fixed, stained, and washed according to the instructions of the Alizarin Red S staining kit (Solarbio, China). Based on the previously reported methods, the stained mineralization nodules were dissolved with $10 \%$ cetylpyridinium chloride at $37^{\circ} \mathrm{C}$ for $30 \mathrm{~min} 22$. The solution was added to a 96 -well plate, and the OD value was measured at $562 \mathrm{~nm}$ for quantitative analysis.

\section{Adipocyte differentiation and Oil red 0 (ORO) staining}

The rBMSCs and MC3T3-E1 cells were seeded into 12-well plates and transfected. When the confluence point of cells reached $100 \%$, the growth medium was changed as the adipocyte differentiation medium (Cyagen, USA). After cultured in adipocyte differentiation medium for 4 weeks, the rBMSCs and MC3T3E1 cells were stained with an Oil red 0 staining kit (Solarbio, China) and pictured using an inverted microscope. Based on the previously reported methods, the stained lipid deposits were extracted with isopropyl alcohol at room temperature for $30 \mathrm{~min} 23$. Finally, the solution was added to a 96-well plate, and the $O D$ value was measured at $562 \mathrm{~nm}$ for quantitative analysis.

\section{The extraction of proteins and Western blot}

Protein lysate (RIPA, Beyotime, Jiangsu, China) and phosphatase inhibitor (cocktail, Abcam, U.S.A.) were added to the cells and bone powder to extract total protein. The protein concentration of the samples was detected using a BCA protein concentration determination kit (Beyotime, China). A total of $50 \mu \mathrm{g}$ protein was loaded into each lane, separated with 10\% SDS-PAGE separation gel (Epizyme Biotech, China) at 60 $V$ for 35 minutes and at $100 \mathrm{~V}$ for about 70 minutes, then transferred to PVDF membrane (Millipore, USA), and closed with $5 \%$ nonfat milk in TBST buffer (CST, U.S.A.). Then, the protein bands were incubated in primary antibodies on a shaking table at $4{ }^{\circ} \mathrm{C}$ overnight. The primary antibodies used in this experiment were as follows: $\beta$-actin and OCN were purchased from Affinity (U.S.A.); Runx2 and FAK were purchased from Wanlei Bio (China); ALP was purchased from Abcam (U.S.A.); PPARy, ERK1/2, p-ERK1/2, p-FAK were purchased from CST (U.S.A.), ITGB8 was purchased from Zenbio (China). After the application, the protein bands were washed with $1 \triangle T B S T$ three times and then incubated with secondary antibodies (Goat anti-rabbit, 1:8,000, biosharp, China) for 1 hour. Then the bands were washed with 1囚TBST three times. Finally, the protein was detected with an ECL reagent (Zen-Bio, China).

\section{Dual-luciferase reporter assay}

Targetscan 7.2 (www.targetscan.org) was used to predict the relationship between the ITGB8 and miR199a-3p. Then, HEK-293T cells were cultured in H/DMEM supplied with 10\% FBS (Gibco, UK), 100 U/mL 
penicillin(NCM, China), and $100 \mu \mathrm{g} / \mathrm{mL}$ streptomycin (NCM, China) at $5 \% \mathrm{CO} 2$ and $37^{\circ} \mathrm{C}$. The miR-199a-3p binding site containing the ITGB8-3'UTR (3511-3530: 5'- CTATGTGTCTTACTACTGTT -3', 4021-4040: GTGCTAAGTTACTACTGCCG) was inserted into the GP-miRGLO vector (GenePharma). A cDNA fragment of the mutant sequence (3511-3530: 5'- CTTACACTCTTTGATGACTT -3', 4021-4040: 5'-

GTGCTAAGTTTGATGACCCG-3') of ITGB8-3'-UTR with a target region was also inserted into the GPmiRGLO vector. The sequence of wild type and mutant type ITGB8 (MT-ITGB8) were further confirmed by sequencing. The co-transfection of WT-ITGB8 or MT-ITGB8 with agomiR-199a-3p or NC was finished using EndofectinTM-MAX reagent in the 96-well plate, respectively. Relative luciferase activity was further detected with the Dual-Luciferase reporter gene detection system (Promega).

\section{Animal study}

All experimental and animal care procedures were approved by the Research Ethics Committee of the Affiliated Hospital of Chongqing Medical University and performed following the guidelines of the National Institutes of Health Guidelines for the Care and Use of Laboratory Animals. A total of 16 female SD rats (8-week old, $180-200 \mathrm{~g}$ ) were enrolled in this study and randomly divided into 4 groups: the control rats $(n=4), M P S$ rats $(n=4)$, and MPS+ NC rats $(n=4)$, MPS+antagomir-199a-3p $(n=4)$ respectively injected with PBS, MPS, MPS+ NC and MPS+antagomir-199a-3p. The MPS was intramuscularly injected into rats and the NC and antagomir-199a-3p were injected into rats via periosteum. Based on the previous studies, intramuscular injections of $20 \mathrm{mg} / \mathrm{kg}$ tetracycline (Solarbio), $10 \mathrm{mg} / \mathrm{kg}$ calcein (Solarbio) and $30 \mathrm{mg} / \mathrm{kg}$ Alizarin red S (Solarbio) were performed at weeks 0, 2, and 4 during the experiment for fluorescence staining24-25. After injected for 6 weeks, all rats were sacrificed to harvest the femoral heads. Then the femoral heads were fixed in $4 \%$ paraformaldehyde and scanned by micro-Computed Tomography (microCT) and analyzed by immunohistochemistry and histology.

A micro-CT (Skyscan1174 X-Ray Microtomography, Bruker, Belgium) was used to scan the rat femoral heads. After scanning, software N-Recon was used for 3-dimensional reconstruction of the femoral heads, and software CT-AN was used to analyze the osteogenic parameters including BV/TV (bone volume per tissue volume), Tb.Sp (trabecular separation), Tb.Th (trabecular thickness) and Tb. N (trabecular number).

\section{Histological analyses and immunohistochemistry (IHC)}

The collected femoral heads were fixed with $4 \%$ paraformaldehyde for a week and decalcified with EDTA decalcifying solution. The H\&E staining was finished and pictured using an optical microscope to detect the effect of miR-199a-3p on rat femoral heads. The expression of RUNX2 and CD31 in bone tissue was measured using $\mathrm{IHC}$ staining.

\section{Immunofluorescence staining}

The femoral heads were fixed in $4 \%$ formaldehyde for $72 \mathrm{~h}$, dehydrated with a gradient of ethanol, and ether for $24 \mathrm{~h}$ at each step. The specimens were infiltrated with polymethylmethacrylate and then 
embedded. Then, $10 \mu \mathrm{m}$ thick sections were made with a Leica hard tissue slicer. The images of the fluorescent-labeled specimens were captured using a confocal laser scanning microscope (Leica, Heidelberg, Germany). The excitation/emission wavelengths were set as follows: 405/560-590 nm (tetracycline, blue), 543/580-670 nm (alizarin red, red), and 488/500-550 nm (calcium green, green).

\section{Statistical analysis}

Statistical analyses were performed using GraphPad PRISM8.0. All of the measurement data were expressed as means \pm standard deviation (SD). For the comparison between two groups, the Student's Ttest method was used, while for the comparison among multiple groups, the one-way ANOVA and Tukey test methods were used. P-value $\leq 0.05$ was considered statistically significant.

\section{Results}

\section{MiR-199a-3p may be involved in the pathological process of SANFH.}

The hip joint X-ray and CT of the SANFH group and FNF group showed the necrosis, collapse, and deformation of the femoral head (Fig.1 A, B). Figure 1C showed sectional images of femoral head specimens from the femoral neck fracture (FNF) group and SANFH group. The H\&E staining showed normal bone trabecula in the FNF group and collapse, disorder, and necrosis of trabecula in the SANFH group (Fig.1D). IHC staining demonstrated that RUNX2 expression was notably declined in necrotic bone tissues, while PPARy was upregulated. (Fig. 1E, F). RT-PCR showed that miR-199a-3p expression was upregulated in necrotic bone tissues and rBMSCs and MC3T3-E1 cells treated with 20UM dexamethasone (Fig. 1G, H, I). All of these data showed that miR-199a-3p may be involved in the pathological process of SANFH.

\section{MiR-199a-3p inhibited osteogenic differentiation of rBMSCs and MC3T3-E1 cells}

To investigate the effects of miR-199a-3p on osteogenesis and adipogenesis of rBMSCs and MC3T3-E1 cells, we transfected the NC, agomiR-199a-3p and antagomir-199a-3p into rBMSCs and MC3T3-E1 cells. We found that the transfection of agomiR-199a-3p and antagomiR-199a-3p could separately increase and decrease the expression of miR-199a-3p in rBMSCs (Fig. 2A) and MC3T3-E1 cells (Fig. 2C). Then, we examined the osteogenic and adipogenic markers and the results of western blotting showed that expression of OCN, ALP, and RUNX2 were declined in the the agomiR-199a-3p group, while the expression of PPARy was increased (Fig. 2B, D). In addition, ALP activity demonstrated the suppressing effect of miR-199a-3p on the ALP activity of rBMSCs and MC3T3-E1 cells (Fig. 2E, F). Alizarin red S staining also exhibited a significant decrease of mineralization on the surface of rBMSCs and MC3T3-E1 cells in the agomiR-199a-3p group (Fig. $2 \mathrm{G}, \mathrm{H}, \mathrm{K}, \mathrm{L}$ ). The Oil red $\mathrm{O}$ staining showed that the transfection of agomiR$199 a-3 p$ enhanced the formation of lipid droplets in rBMSCs, compared with those in the NC group and antagomir-199a-3p group (Fig. 2H, I, M, N). These data indicate that miR-199a-3p could inhibit the osteogenic differentiation of rBMSCs and MC3T3-E1 cells, enhancing adipogenic differentiation. 


\section{Integrin $\beta 8$ (ITGB8) was the direct target of miR-199a-3p}

We found that there were two binding sites between miR-199a-3p and ITGB8 (Fig. 3A). Next, we constructed WT-ITGB8 and MT-ITGB8 plasmids (Fig. 3A) and conducted a dual-luciferase reporter assay. The results of the dual-luciferase reporter assay showed that the overexpression of miR-199a-3p significantly suppressed the luciferase activity of 3'-UTR in the WT-ITGB8 group, whereas no differences in luciferase activity of 3'-UTR were observed in the MT-ITGB8 group (Fig. 3B). These results indicated that miR-199a-3p could directly target and bind to ITGB8. Furthermore, the results of western blotting proved that ITGB8 expression was significantly reduced by force expression of miR-199a-3p in rBMSCs and MC3T3-E1 cells (Fig. 3C, D). The IHC staining also showed that ITGB8 was reduced in SANFH patients (Fig.3E). The above results confirmed that ITGB8 was the target gene of miR-199a-3p.'

\section{MiR-199a-3p inhibited osteogenesis of rBMSCs and MC3T3-E1 cells by down-regulating ITGB8 and inactivating the ITGB8-FAK-ERK1/2-RUNX2 axis}

To further investigate the function of ITGB8, we silenced the expression of ITGB8 to study changes in osteogenic differentiation of rBMSCs and MC3T3-E1 cells. The silence of ITGB8 significantly inhibited osteogenic differentiation of rBMSCs and MC3T3-E1 cells, evidenced through downregulated osteogenic markers (Fig. 4A, B), reduced ALP activity (Fig. 4C, D), and decreased calcium deposits (Fig. 4E-H). We also found that the expression of PPARy and the lipid droplets formation were negatively related to the expression of ITGB8 (Fig. 4A, B, I-L). In addition, the inhibition of miR-199a-3p (antagomiR-199a-3p) partly rescued the imbalance between osteogenic differentiation and adipogenic differentiation of rBMSCs and MC3T3-E1 cells induced by the interference of ITGB8.

From the KEGG database, we found that we found that integrin $\beta 8$ was associated with the focal adhesion pathway. In this pathway, integrins phosphorylate the focal adhesion kinase (FAK) and activate it, p-FAK activates the Erk1/2, and then p-Erk1/2 interacts with RUNX2 and promote osteogenesis. According to the previous studies, the Erk1/2-RUNX2 pathway was closely associated with osteogenesis26-28. Therefore, we assumed that miR-199a-3p suppressed the osteogenic differentiation of rBMSCs and MC3T3-E1 cells by down-regulating the integrin B8-FAK-Erk1/2-Runx2 pathway. We next detected ITGB8, FAK, p-FAK, Erk1/2, p-Erk1/2, and RUNX2(Fig. 4M, N). It was obvious that silTGB8 influenced the expression of these proteins in rBMSCs and MC3T3-E1 cells compared with the NC group. However, The suppression of miR-199a-3p partly resolved the impairment of the ITGB8-FAK-Erk1/2RUNX2 pathway caused by silTGB8. Taken together, these data suggest that the ITGB8-FAK-Erk1/2RUNX2 pathway is involved in the osteogenesis of rBMSCs and MC3T3-E1 cells through interaction with miR-199a-3p.

\section{Silencing of miR-199-3p rescued the suppression of osteogenesis of rBMSCs and MC3T3-E1 cells induced by dexamethasone}

The experiments in figure 4 illustrated that antagomiR-199a-3p remedied the decreased osteogenesis of rBMSCs and MC3T3-E1 cells caused by inhibition of ITGB8. Then we investigated the effect of 
antagomiR-199a-3p (silence of miR-199a-3p) on glucocorticoid-induced impairment in rBMSCs and MC3T3-E1 cells. RT-PCR revealed that dexamethasone (DEX) could lead to the upregulation of miR-199a$3 p$ in rBMSCs and MC3T3-E1 cells and the upregulation was rescued by the transfection of antagomiR199a-3p (Fig. 5 A, C). After treated with dexamethasone for 3 days, the expression of OCN, ALP, ITGB8, pFAK, p-Erk1/2, and RUNX2 were dramatically decreased with the augment of PPARY (Fig. 5B, D). However, silencing antagomiR-199a-3p antagonized the above-mentioned trend. In addition, the ALP activity, Alizarin red S staining showed that the silence of antagomiR-199a-3p could restore the reduced ALP activity and mineralization of rBMSCs and MC3T3-E1 cells caused by dexamethasone antagomiR-199a3p (Fig. 5 E-H, K-L). Oil red O staining demonstrated a similar protective effect of antagomiR-199a-3p (Fig. $5 \mathrm{I}-\mathrm{J}, \mathrm{M}-\mathrm{N}$ ). These data suggested that antagomiR-199a-3p could remedy the damage of glucocorticoids on the differentiation of rBMSCs and MC3T3-E1 cells.

\section{Silencing miR-199a-3p protect GC-induced SANFH damage in vivo}

Finally, antagomiR-199a-3p was injected into rats to observe its protective role against glucocorticoids in vivo (Fig. 6 A). Micro-CT scanning was conducted to evaluate the bone tissues of the rat femoral head. The results showed that the SANFH models were successfully conducted by the injection of methylprednisolone (MPS) and the collapse of the femoral was observed. By contrast, the treatment of antagomiR-199a-3p remarkably attenuated the pathological changes of SANFH (Fig. 6 B). In addition, MPS injection led to serious deterioration of trabecular parameters, such as BV/TV, Tb. N, Tb.Th and Tb.Sp. However, the silence of miR-199a-3p increased these parameters (Fig. 6C). The results of the H\&E staining showed that there was more trabecular bone structure and less empty lacunae in the femoral head of the MPS+antagomiR-199a-3p group compared to the MPS+NC group (Fig. 6D, E). The IHC staining showed that ITGB8, RUNX2 were downregulated in the MPS group, while PPARY was upregulated (Fig. 6F, G, H). The expression of ITGB8, RUNX2, and PPARy was significantly restored by the silence of miR-199a-3p. Overall, these data suggested that antagomiR-199a-3p were able to treat GC-induced SANFH damage in vivo.

The dynamic bone formation and mineralization in the femoral head were displayed using fluorochrome including tetracycline, Alizarin red S, and Calcein. The femoral head in the control group had a stronger fluorochrome labeling with tetracycline (blue), alizarin red S (red), and calcein (green) in the femoral head. However, the femoral head in the MPS group and MPS+NC group had significantly decreased new bone formation evidenced by much weaker fluorochrome labeling in the femoral head (Figure 7). On the contrary, a much broader area of subchondral trabeculae was stained by fluorochrome labeling in the MPS + antagomiR-199a-3p group, indicating the beneficial effect of antagomir-199a-3p on steroidinduced avascular necrosis of the femoral head (Figure 7).

\section{Discussion}

Resulted from the wide usage of glucocorticoids (GCs), SANFH has brought tremendous pressure to society 1 . The detailed mechanisms involved in the initiation and development of SANFH are complicated 
and remain unclear. Recently, considerable studies have revealed that a high concentration of GCs could inhibit osteogenesis and promote adipogenesis29-31. In the present study, we observed that the miR$199 a-3 p$ was dysregulated in vitro and in vivo. Moreover, we revealed the key role of the miR-199a-3p in mediating osteogenesis and adipogenesis of rBMSCs and MC3T3-E1 cells and provided fruitful and useful underlying targets to prevent the development of SANFH. In our study, we confirmed the harmful role in SANFH and identified new target molecules and the mechanisms by which miR-199a-3p suppressed osteogenesis and promoted adipogenesis.

Many differentially expressed miRNAs were identified in SANFH and the broad biological significance importance of miRNAs on osteogenesis and adipogenesis of SANFH has been investigated, including miR-155-5p16, miR-70832, miR-206-3p33, miR-26a34, and miR-144-3p35. The downstream target genes of these miRNAs include GSK3B, SAMD3, connexin43, EZH2, and FZD4. However, the role of miR-199a-3p in SANFH has never been studied before. In the present study, we first found that in the necrotic bone tissues of patients with SANFH, the osteogenic marker was reduced while miR-199a-3p and PPARy were notably up-regulated. To understand the role of miR-199a-3p in the pathogenesis of SANFH, NC, agomiR199a-3p, and antagomir-199a-3p were transfected into rBMSCs and MC3T3-E1 cells to detect their effects on osteogenesis and adipogenesis. The data indicated that miR-199a-3p was negatively connected with osteogenesis and positively connected with adipogenesis. Moreover, it was observed that the silencing of miR-199a-3p could significantly reverse the impairment caused by glucocorticoids in vitro and in vivo. Thus, our findings provide new insight into the regulation of osteogenesis, adipogenesis, and progression of SANFH.

To study the molecular mechanism by which miR-199a-3p regulates the differentiation of rBMSCs and MC3T3-E1 cells, we performed a search with TargetScan, which revealed that ITGB8 might be a possible target with 2 sites complementary to miR-199a-3p in its 3"UTR. To confirm this prediction, we conducted a dual-luciferase reporter assay and identified ITGB8 as a direct target of miR-199a-3p. Moreover, WB showed that upregulation of miR-199a-3p expression led to downregulation of ITGB8 at the protein level, whereas functional inhibition of miR-199a-3p led to derepression of ITGB8, strongly suggesting that ITGB8 is regulated by miR-199a-3p during osteogenic differentiation and adipogenic differentiation of rBMSCs and MC3T3-E1 cells. In addition, the silence of silencing of ITGB8 expression inhibited osteogenesis and angiogenesis, while these effects were rescued by antagomiR-199a-3p. ITGB8, also named integrin $\beta 8$, is an important member of the integrin family. Previously, there was little research focused on the role of ITGB8 in osteogenesis and adipogenesis. It has been proved that the expression of ITGB8 was upregulated during the osteogenic differentiation of MSCs into osteoblasts and declined during adipogenesis36,37. However, no researcher conduct specific to explore if ITGB8 could regulate osteoblastic differentiation and adipogenic differentiation as well as the role of ITGB8 in SANFH. In our study, our study first proved that ITGB8 declined in necrotic bone tissues of patients with SANFH. In addition, we first found that ITGB8 could regulate osteogenic differentiation of rBMSCs and MC3T3-E1 cells by interacting with the FAK-Erk1/2-RUNX2 pathway and proved the central role of the miR-199a-3pITGB8-FAK-Erk1/2-RUNX2 axis in SANFH. Moreover, it is believed that GCs could suppress the 
osteogenesis and promote adipogenesis by miR-199a-3p targeting ITGB8 and inactivating the ITGB8FAK-Erk1/2-RUNX2 pathway.

Several studies have reported the direct use of miRNAs in the treatment of SANFH. Zuo et al. reported that miR-26a overexpressed in CD34+ stem cell-derived exosomes could protect the femoral head from damage caused by GCs by strengthening angiogenesis and osteogenesis 38 . Cao et al. reported that miR224-5p silencing protected against GC-induced reductions of SMAD4 and promoted osteogenesis to alleviate SANFH in vitro and in vivo39. In this study, we directly used antagomir-199a-3p in rats and the utility of antagomiR-199a-3p could improve new bone formation in the rat femoral head, which could reduce the degree and incidence of SANFH. Moreover, Calcein and Alizarin Red S which could be absorbed and deposited in bone tissues were used to show the dynamic bone formation and the results showed that antagomir-199a-3p helped bone formation and mineralization and alleviate the harmful effect of glucocorticoids in vivo.

\section{Conclusion}

Taken together, these data highlighted that the upregulation of miR-199a-3p induced by GCs could reciprocally regulate the adipo-osteogenic differentiation of rBMSCs and MC3T3-E1 cells by targeting ITGB8. The miR-199a-3p-ITGB8-FAK-Erk1/2-RUNX2 axis is a potential signaling mechanism, and inhibition of miR-199a-3p expression could promote osteogenesis and inhibit adipogenesis to alleviate the occurrence of SANFH, which is a novel therapeutic target for the treatment of SAFH.

\section{Declarations}

\section{Acknowledgements}

We appreciate the Key Laboratory of Diagnostic Medicine Designated by the Ministry of Education, Chongqing Medical University for providing experimental platform.

\section{Authors' contributions}

Conception and design of the experiments: Wu Yang, Tingmei Chen, Jian Zhang. Methodology: Wu Yang, Yunfei Yang, Minkang Guo. Collection, analysis and interpretation of all data: Wu Yang, Yunfei Yang, Weiwen Zhu. Resources: Wu Yang, Minkang Guo, Husun Qian. Writing the original manuscript: Wu Yang. Review \& Editing the manuscript: Wu Yang, Chengjie Lian, Haobo Bai, Jian Zhang. Funding acquisition and project administration: Jian Zhang, Chengjie Lian. All authors read and approved the final manuscript.

\section{Funding}

This work was funded by the Medical Research Project of Health and Family Planning Commission in Chongqing (Grant No. 2017ZDXM006), the General Project of Technology Innovation and Application 
Development of Chongqing Science and Technology Bureau (Grant No. cstc2019jscx-msxmX0245), the Natural Science Foundation of Chongqing Science and Technology Commission (No. cstc2020jcyjmsxmX0179) and the First Affiliated Hospital of Chongqing Medical University cultivating fund (PYJJ2019-203).

\section{Availability of data and materials}

The data presented in this study are available on request from the corresponding author. The data are not publicly available due to the non-completion of the clinical study.

\section{Declarations}

\section{Ethics approval and consent to participate}

This study was approved by the Research Ethics Committee of The Affiliated Hospital of Chongqing Medical University. Informed consent was obtained from all subjects involved in the study. All animal studies complied with the principles based on the International Guiding Principles for Biomedical Research Involving Animals.

\section{Consent to publish}

Not applicable.

\section{Competing interests}

The authors declare that they have no competing interests.

\section{References}

1. van der Jagt D, Mokete L, Pietrzak J, Zalavras CG, Lieberman JR. Osteonecrosis of the femoral head: evaluation and treatment. J Am Acad Orthop Surg. 2015;23(2):69-70. doi: 10.5435/JAAOS-D-1400431. PMID: 25624358.

2. Mont MA, Cherian JJ, Sierra RJ, et al. Nontraumatic Osteonecrosis of the Femoral Head: Where Do We Stand Today? A Ten-Year Update. J Bone Joint Surg Am. 2015;97(19):1604-27. doi: 10.2106/JBJS.0.00071. PMID: 26446969.

3. Chang C, Greenspan A, Gershwin ME. The pathogenesis, diagnosis and clinical manifestations of steroid-induced osteonecrosis. J Autoimmun. 2020;110:102460. doi: 10.1016/j.jaut.2020.102460. PMID: 32307211.

4. Lee YJ, Cui Q, Koo KH. Is There a Role of Pharmacological Treatments in the Prevention or Treatment of Osteonecrosis of the Femoral Head?: A Systematic Review. J Bone Metab. 2019 Feb;26(1):13-18. doi: 10.11005/jbm.2019.26.1.13. PMID: 30899719. 
5. Zhu W, Guo M, Yang W, et al. CD41-deficient exosomes from non-traumatic femoral head necrosis tissues impair osteogenic differentiation and migration of mesenchymal stem cells. Cell Death Dis. 2020;11(4):293. doi: 10.1038/s41419-020-2496-y. PMID: 32341357.

6. Mont MA, Salem HS, Piuzzi NS, et al. Nontraumatic Osteonecrosis of the Femoral Head: Where Do We Stand Today?: A 5-Year Update. J Bone Joint Surg Am. 2020;102(12):1084-1099. doi: 10.2106/JBJS.19.01271. PMID: 32282421.

7. Mont MA, Cherian JJ, Sierra RJ, Jones LC, Lieberman JR. Nontraumatic Osteonecrosis of the Femoral Head: Where Do We Stand Today? A Ten-Year Update. J Bone Joint Surg Am. 2015 Oct 7;97(19):1604-27. doi: 10.2106/JBJS.0.00071. PMID: 26446969.

8. Houdek MT, Wyles CC, Packard BD, et al. Decreased Osteogenic Activity of Mesenchymal Stem Cells in Patients With Corticosteroid-Induced Osteonecrosis of the Femoral Head. J Arthroplasty. doi: 10.1016/j.arth.2015.08.017. PMID: 26404846.

9. Bianco P. "Mesenchymal" stem cells. Annu Rev Cell Dev Biol. 2014;30:677-704. doi: 10.1146/annurevcellbio-100913-013132. PMID: 25150008.

10. Wang BL, Sun W, Shi ZC, et al. Decreased proliferation of mesenchymal stem cells in corticosteroidinduced osteonecrosis of femoral head. Orthopedics. 2008;31(5):444. doi: 10.3928/0147744720080501-33. PMID: 19292322.

11. Ambros V. The functions of animal microRNAs. Nature. 2004 Sep 16;431(7006):350-5. doi: 10.1038/nature02871. PMID: 15372042.

12. O'Brien J, Hayder H, Zayed Y, et al. Overview of MicroRNA Biogenesis, Mechanisms of Actions, and Circulation. Front Endocrinol (Lausanne). 2018;9:402. doi: 10.3389/fendo.2018.00402. PMID: 30123182.

13. Fang $\mathrm{SH}$, Chen $\mathrm{L}$, Chen HH, et al. MiR-15b ameliorates SONFH by targeting Smad7 and inhibiting osteogenic differentiation of BMSCs. Eur Rev Med Pharmacol Sci. 2019 Nov;23(22):9761-9771. doi: 10.26355/eurrev_201911_19539. PMID: 31799643.

14. Duan DY, Tang J, Tian HT, et al. Adipocyte-secreted microvesicle-derived miR-148a regulates adipogenic and osteogenic differentiation by targeting Wnt5a/Ror2 pathway. Life Sci. 2021;278:119548. doi: 10.1016/j.Ifs.2021.119548. PMID: 33930365.

15. Kong L, Zuo R, Wang M, et al. Silencing MicroRNA-137-3p, which Targets RUNX2 and CXCL12 Prevents Steroid-induced Osteonecrosis of the Femoral Head by Facilitating Osteogenesis and Angiogenesis. Int J Biol Sci. 2020;16(4):655-670. doi: 10.7150/ijbs.38713. PMID: 32025213.

16. Wu F, Huang W, Yang Y, et al. miR-155-5p regulates mesenchymal stem cell osteogenesis and proliferation by targeting GSK3B in steroid-associated osteonecrosis. Cell Biol Int. 2021;45(1):83-91. doi: 10.1002/cbin.11470. PMID: 32991030.

17. Wu JC, Sun J, Xu JC, et al. Down-regulated microRNA-199a-3p enhances osteogenic differentiation of bone marrow mesenchymal stem cells by targeting $\mathrm{Kdm} 3 \mathrm{a}$ in ovariectomized rats. Biochem $\mathrm{J}$. 2021;478(4):721-734. doi: 10.1042/BCJ20200314. PMID: 33410908. 
18. Rasheed Z, Rasheed N, Al-Shobaili HA. Epigallocatechin-3-O-gallate up-regulates microRNA-199a-3p expression by down-regulating the expression of cyclooxygenase-2 in stimulated human osteoarthritis chondrocytes. J Cell Mol Med. 2016;20(12):2241-2248. doi: 10.1111/jcmm.12897.. PMID: 27515563.

19. Wangyang Y, Yi L, Wang T, et al. MiR-199a-3p inhibits proliferation and induces apoptosis in rheumatoid arthritis fibroblast-like synoviocytes via suppressing retinoblastoma 1. Biosci Rep. 2018;38(6):BSR20180982. doi: 10.1042/BSR20180982. PMID: 30352835.

20. Zhao J, Liu Z, Chang Z. Osteogenic differentiation and calcification of human aortic smooth muscle cells is induced by the RCN2/STAT3/miR-155-5p feedback loop. Vascul Pharmacol. 2021;136:106821. doi: 10.1016/j.vph.2020.106821. PMID: 33221530.

21. Chang Z, Yan G, Zheng J, et al. The IncRNA GAS5 Inhibits the Osteogenic Differentiation and Calcification of Human Vascular Smooth Muscle Cells. Calcif Tissue Int. 2020;107(1):86-95. doi: 10.1007/s00223-020-00696-1. PMID: 32347320.

22. Kim HY, Park SY, Choung SY. Enhancing effects of myricetin on the osteogenic differentiation of human periodontal ligament stem cells via BMP-2/Smad and ERK/JNK/p38 mitogen-activated protein kinase signaling pathway. Eur J Pharmacol. 2018;834:84-91. doi:

10.1016/j.ejphar.2018.07.012.

23. Kraus NA, Ehebauer F, Zapp B, et al. Quantitative assessment of adipocyte differentiation in cell culture. Adipocyte. 2016;5(4):351-358. doi: 10.1080/21623945.2016.1240137.

24. Chen YX, Zhu DY, Yin JH, et al. The protective effect of PFTa on alcohol-induced osteonecrosis of the femoral head. Oncotarget. 2017;8(59):100691-100707. doi: 10.18632/oncotarget.19160. PMID: 29246013.

25. Yu H, Liu P, Zhu D, et al. Chrysophanic acid shifts the differentiation tendency of BMSCs to prevent alcohol-induced osteonecrosis of the femoral head. Cell Prolif. 2020;53(8):e12871. doi: 10.1111/cpr.12871. PMID: 32597546.

26. Yuh DY, Maekawa T, Li X, et al. The secreted protein DEL-1 activates a $\beta 3$ integrin-FAK-ERK1/2RUNX2 pathway and promotes osteogenic differentiation and bone regeneration. J Biol Chem. 2020;295(21):7261-7273. doi: 10.1074/jbc.RA120.013024. PMID: 32280065.

27. Ren LR, Yao RB, Wang SY, et al. MiR-27a-3p promotes the osteogenic differentiation by activating CRY2/ERK1/2 axis. Mol Med. 2021;27(1):43. doi: 10.1186/s10020-021-00303-5. PMID: 33902432;

28. Liu L, Zong C, Li B, et al. The interaction between $\beta 1$ integrins and ERK1/2 in osteogenic differentiation of human mesenchymal stem cells under fluid shear stress modelled by a perfusion system. J Tissue Eng Regen Med. 2014;8(2):85-96. doi: 10.1002/term.1498. PMID: 22610905.

29. Chen Q, Shou P, Zheng C, et al. Fate decision of mesenchymal stem cells: adipocytes or osteoblasts? Cell Death Differ. 2016 Jul;23(7):1128-39. doi: 10.1038/cdd.2015.168. PMID: 26868907.

30. Yu RH, Zhang XY, Xu W, et al. Apolipoprotein D alleviates glucocorticoid-induced osteogenesis suppression in bone marrow mesenchymal stem cells via the PI3K/Akt pathway. J Orthop Surg Res. 2020;15(1):307. doi: 10.1186/s13018-020-01824-1. PMID: 32771037. 
31. Chen G, Wang Q, Li Z, et al. Circular RNA CDR1as promotes adipogenic and suppresses osteogenic differentiation of BMSCs in steroid-induced osteonecrosis of the femoral head. Bone. 2020;133:115258. doi: 10.1016/j.bone.2020.115258. PMID: 32018039.

32. Hao C, Yang S, Xu W, et al. MiR-708 promotes steroid-induced osteonecrosis of femoral head, suppresses osteogenic differentiation by targeting SMAD3. Sci Rep. 2016;6:22599. doi: 10.1038/srep22599. PMID: 26932538.

33. Liu G, Luo G, Bo Z, et al. Impaired osteogenic differentiation associated with connexin43/microRNA206 in steroid-induced avascular necrosis of the femoral head. Exp Mol Pathol. 2016;101(1):89-99. doi: 10.1016/j.yexmp.2016.07.009. PMID: 27450649.

34. Li G, Liu H, Zhang X, et al. The protective effects of microRNA-26a in steroid-induced osteonecrosis of the femoral head by repressing EZH2. Cell Cycle. 2020;19(5):551-566. doi:

10.1080/15384101.2020.1717043. PMID: 32054404.

35. Sun Z, Wu F, Yang Y, et al. MiR-144-3p Inhibits BMSC Proliferation and Osteogenic Differentiation Via Targeting FZD4 in Steroid-Associated Osteonecrosis. Curr Pharm Des. 2019;25(45):4806-4812. doi: 10.2174/1381612825666190930094019. PMID: 31566128.

36. Lee HM, Seo SR, Kim J, et al. Expression dynamics of integrin a2, a3, and aV upon osteogenic differentiation of human mesenchymal stem cells. Stem Cell Res Ther. 2020;11(1):210. doi: 10.1186/s13287-020-01714-7. PMID: 32493499.

37. Ullah M, Sittinger M, Ringe J. Extracellular matrix of adipogenically differentiated mesenchymal stem cells reveals a network of collagen filaments, mostly interwoven by hexagonal structural units. Matrix Biol. 2013;32(7-8):452-65. doi: 10.1016/j.matbio.2013.07.001. PMID: 23851162.

38. Zuo R, Kong L, Wang M, et al. Exosomes derived from human CD34+ stem cells transfected with miR-26a prevent glucocorticoid-induced osteonecrosis of the femoral head by promoting angiogenesis and osteogenesis. Stem Cell Res Ther. 2019;10(1):321. doi: 10.1186/s13287-019-14263. PMID: 31730486.

39. Cao Y, Jiang C, Wang X, et al. Reciprocal effect of microRNA-224 on osteogenesis and adipogenesis in steroid-induced osteonecrosis of the femoral head. Bone. 2021;145:115844. doi: 10.1016/j.bone.2021.115844. PMID: 33453444.

\section{Figures}



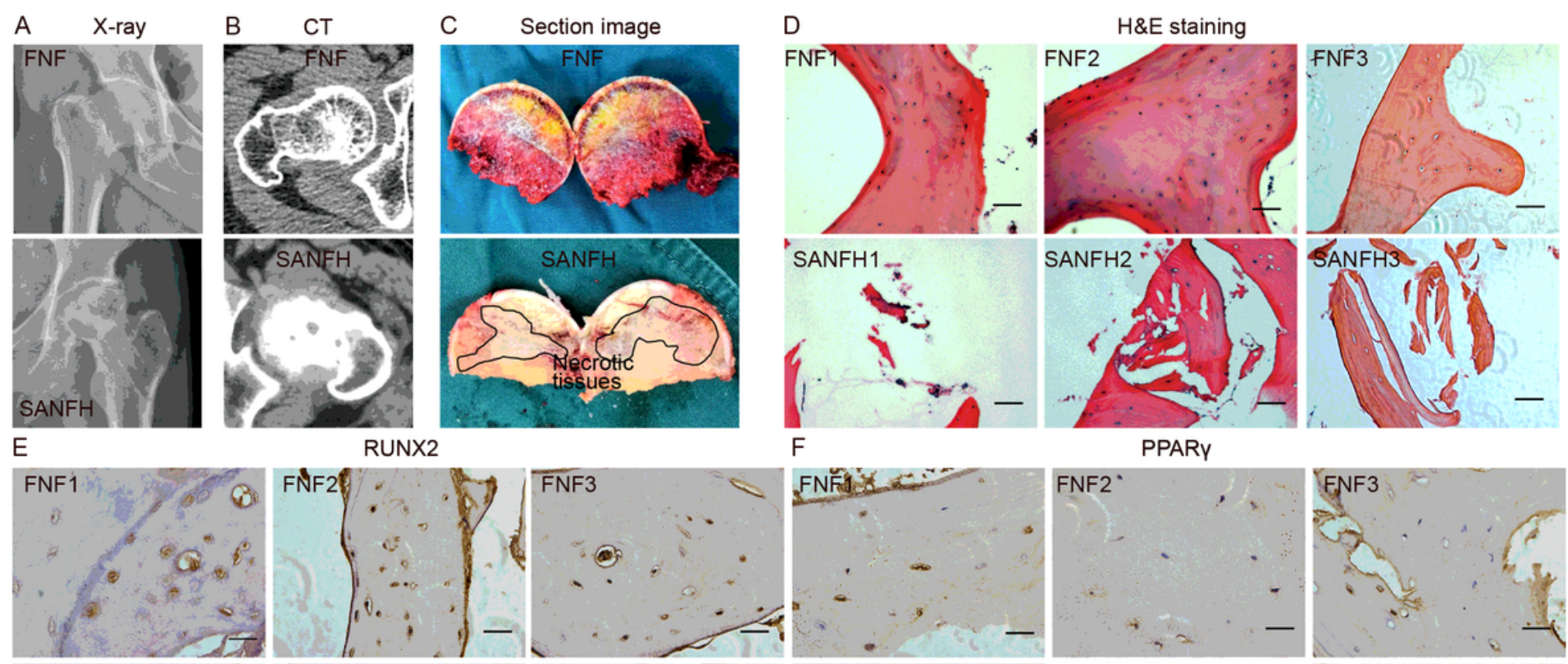

RUNX2

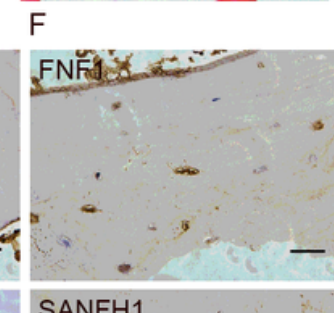

PPARY
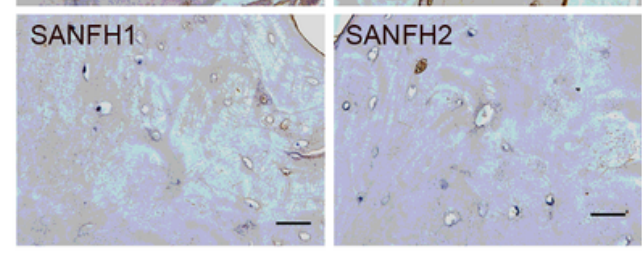

G Bone tissues

$\mathrm{H}$

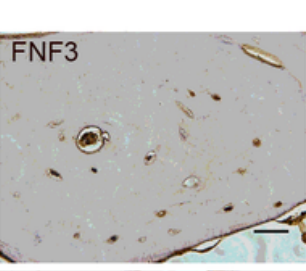

SANFH3
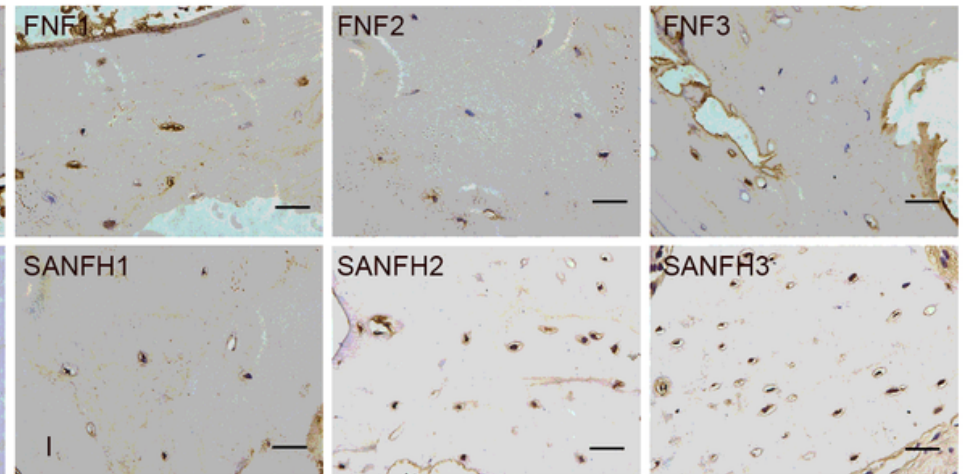

SANFH2

SANFH3:

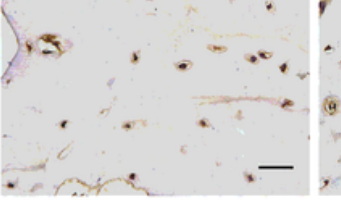

MC3T3-E1
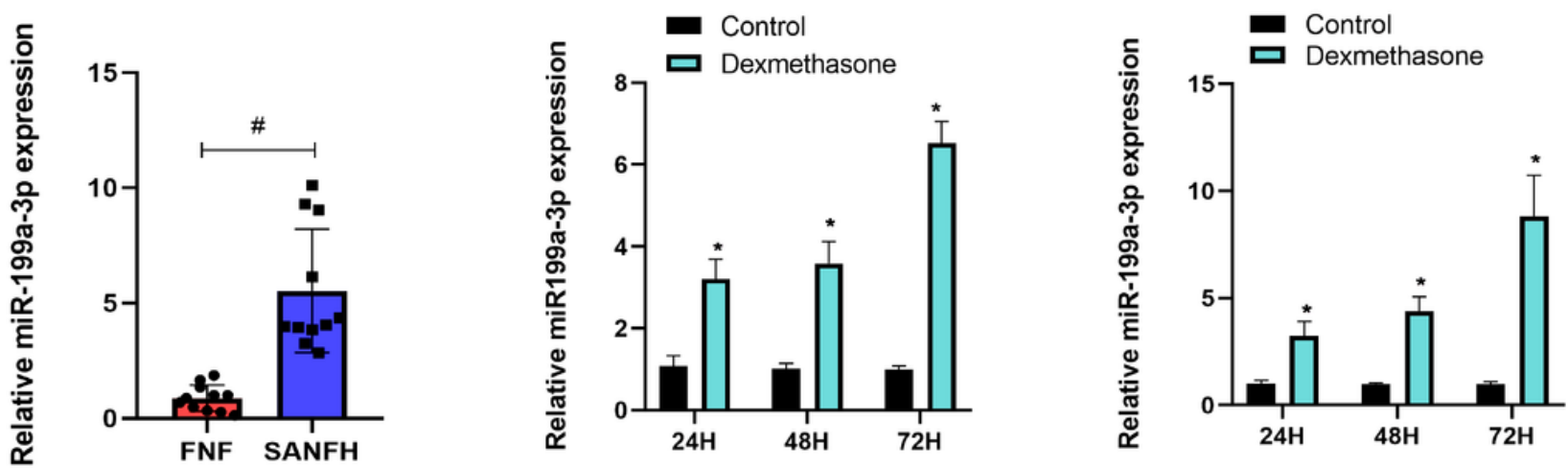

Figure 1

MiR-199a-3p may be involved in the pathological process of SANFH. A-D Representative X-rays (A), CT images (B), sectional images (C), and H\&E staining (D) of femoral heads from FNF and SANFH patients (scale bar= $200 \mu \mathrm{m}$ ). E-F IHC staining was used to detect RUNX2 (E) and PPARY (F) expression in femoral heads of FNF and NONFH patients (scale bar= $50 \mu \mathrm{m}$ ). G-I RT-PCR was used to measure the expression of miR-199a-3p in bone tissues of the FNF group and SANFH group (G), rBMSCs $(\mathrm{H})$, and MC3T3-E1 cells (I) treated with 20 UM dexamethasone. \#\# $P<0.01$, vs. FNF group. * $P<0.05$, vs. control group. DEX: dexamethasone. Data were expressed as mean \pm SD. 

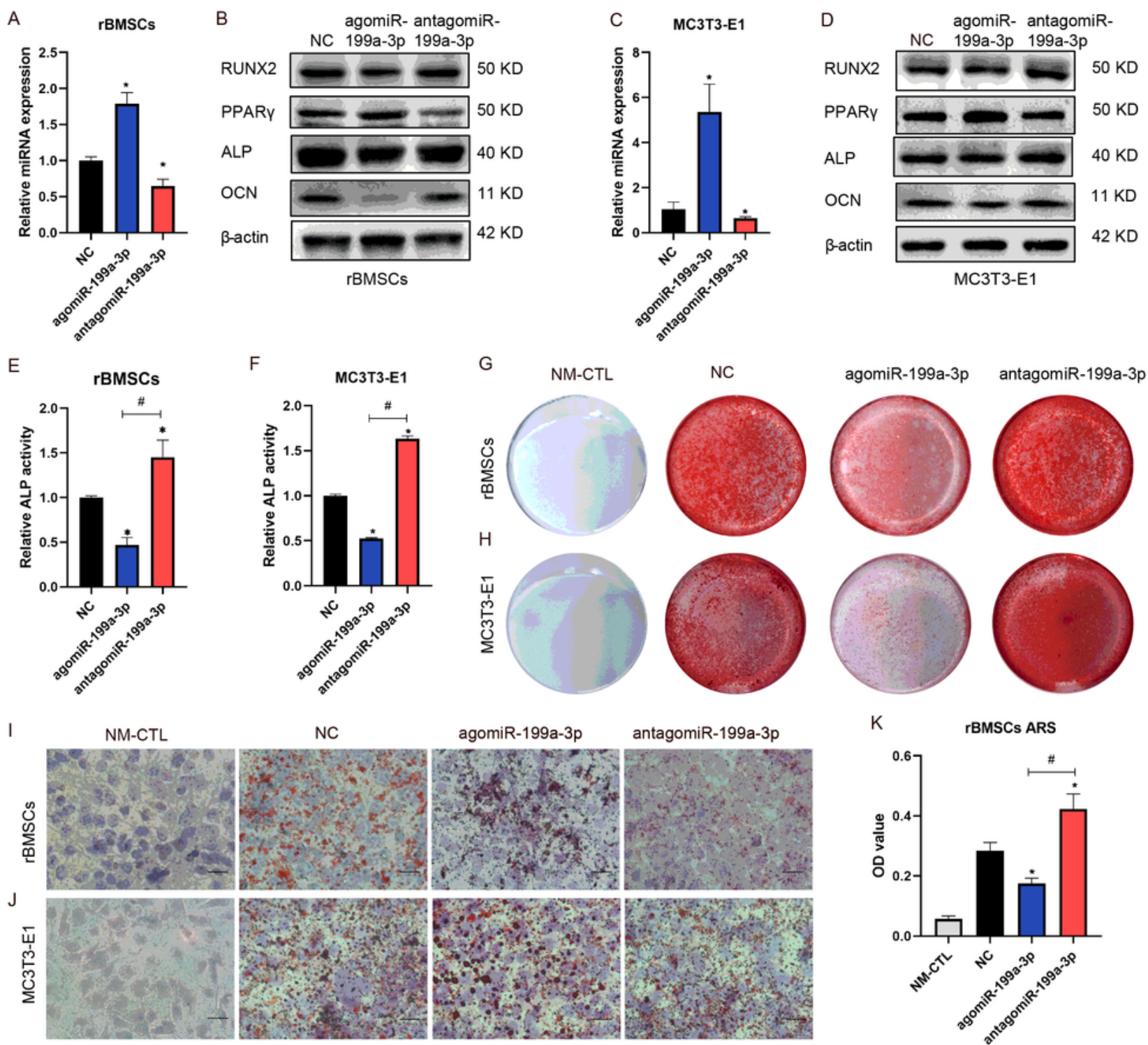

agomiR-199a-3p

antagomiR-199a-3p
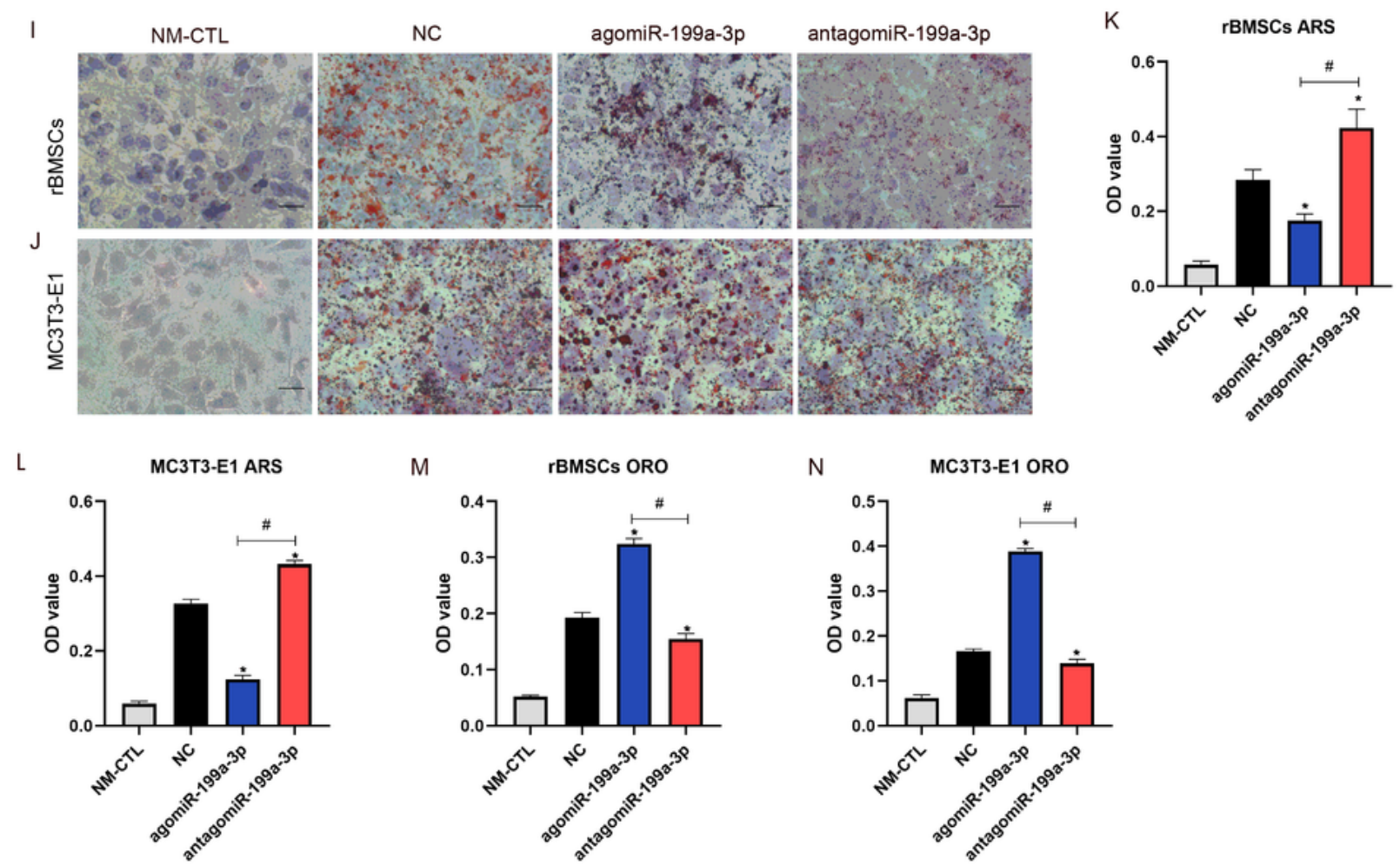

Figure 2

MiR-199a-3p inhibited osteogenic differentiation of rBMSCs and MC3T3-E1 cells. A The expression of miR-199a-3p in rBMSCs after transfected with NC, agomiR-199a-3p and antagomiR-199a-3p. B WB was used to measure the expression of OCN, RUNX2, ALP, and PPARY in rBMSCs. C The expression of miR199a-3p in MC3T3-E1 cells after transfected with NC, agomiR-199a-3p and antagomiR-199a-3p. D WB was used to measure the expression of OCN, RUNX2, ALP, and PPARY in MC3T3-E1 cells. E-F ALP activity 
of rBMSCs (E) and MC3T3-E1 cells (F). G-H Alizarin red S staining of rBMSCs (G) and MC3T3-E1 cells (H) after cultured in ODM for 3 weeks. I-J Oil red O staining of rBMSCs $(\mathrm{I})$ and MC3T3-E1 cells $(\mathrm{J})$ after cultured in adipocyte differentiation medium for 4 weeks (scale bar $=50 \mu \mathrm{m}$ ). K-L quantification of Alizarin red $S$ staining. M-N quantification of Oil red $O$ staining. ${ }^{*} P<0.05$, vs. NC group; $\# P<0.05$, vs. agomiR-199a-3p group. All data were expressed as mean \pm SD.

A

WT - ITGB8 3'UTR 5'- TGAACT ATGTGTCTTACT ACTGTT -3' miR-199a-3p 3'-AUUGGUUACACGU CUGAUGACA -5'

MT - ITGB8 3'UTR 5'- TGAACTTACACTCTT TGA TGACTT -3'

WT - ITGB8 3'UTR 5'- GACTGGTGCTAAGTTACT ACTGCC-3 miR-199a-3p 3'-AUUGGUUACACGUCUGAUGACA -5'

MT - ITGB8 3'UTR 5'-GACTGGTGCTAAGTTTGATGACCC-3'

$\mathrm{E}$

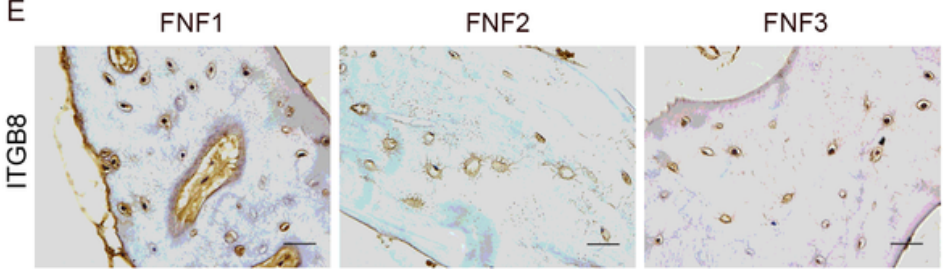

B
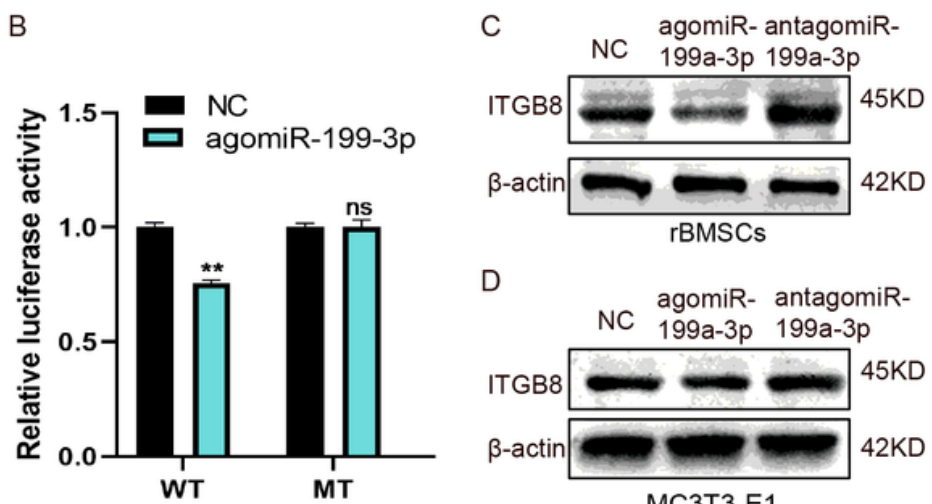

D

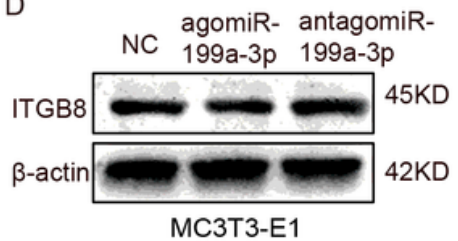

SANFH2

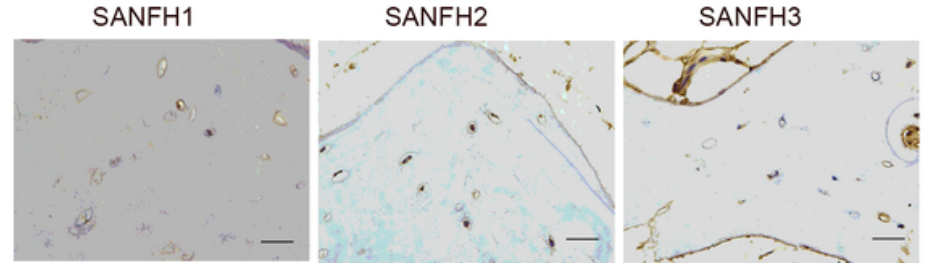

\section{Figure 3}

Integrin $\beta 8$ (ITGB8) was the direct target of miR-199a-3p. A The WT-ITGB8 and MT-ITGB8 plasmids. B The targeting relationship between miR-199a-3p and ITGB8 was verified using a dual luciferase assay. C-D Western blot was conducted to confirm the relationship between miR-199a-3p and ITGB8 in rBMSCs (C) and MC3T3-E1 cells (D). E IHC was used to detect the expression of ITGB8 in bone tissues of patients with FNF or SANFH(scale bar $=50 \mu \mathrm{m})$. ${ }^{*} \mathrm{P}<0.01$, vs. NC group; ns $\mathrm{P} \otimes 0.05$, vs. NC group. All data were expressed as mean \pm SD. 

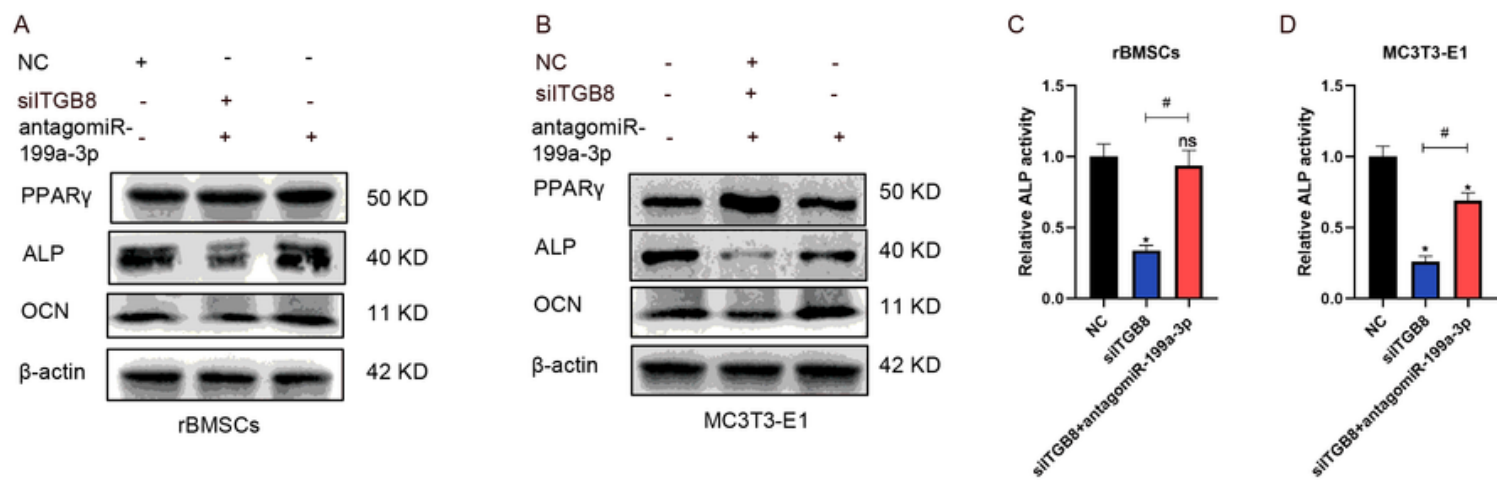

E
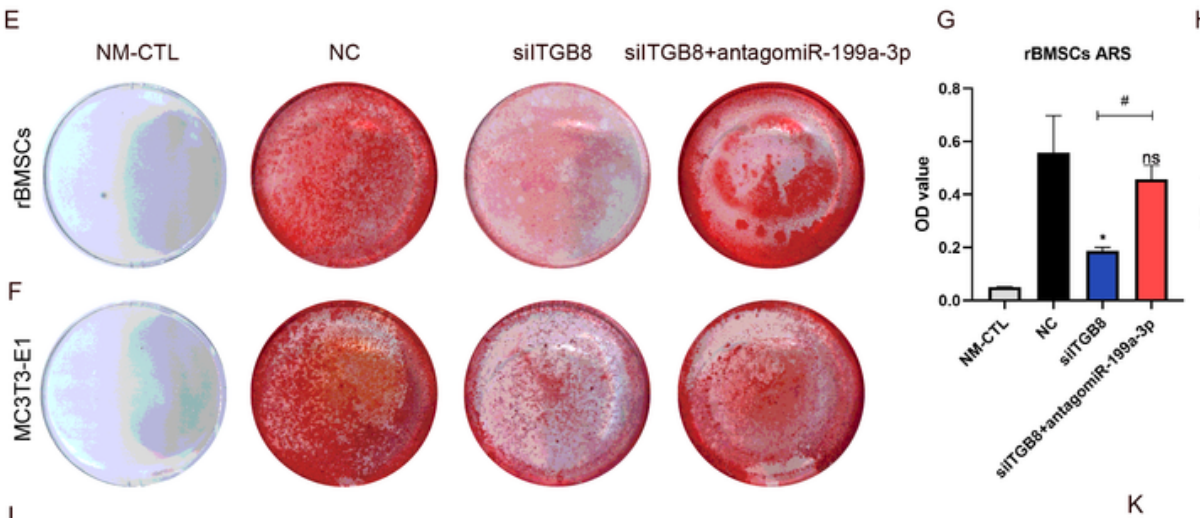

$\mathrm{H}$
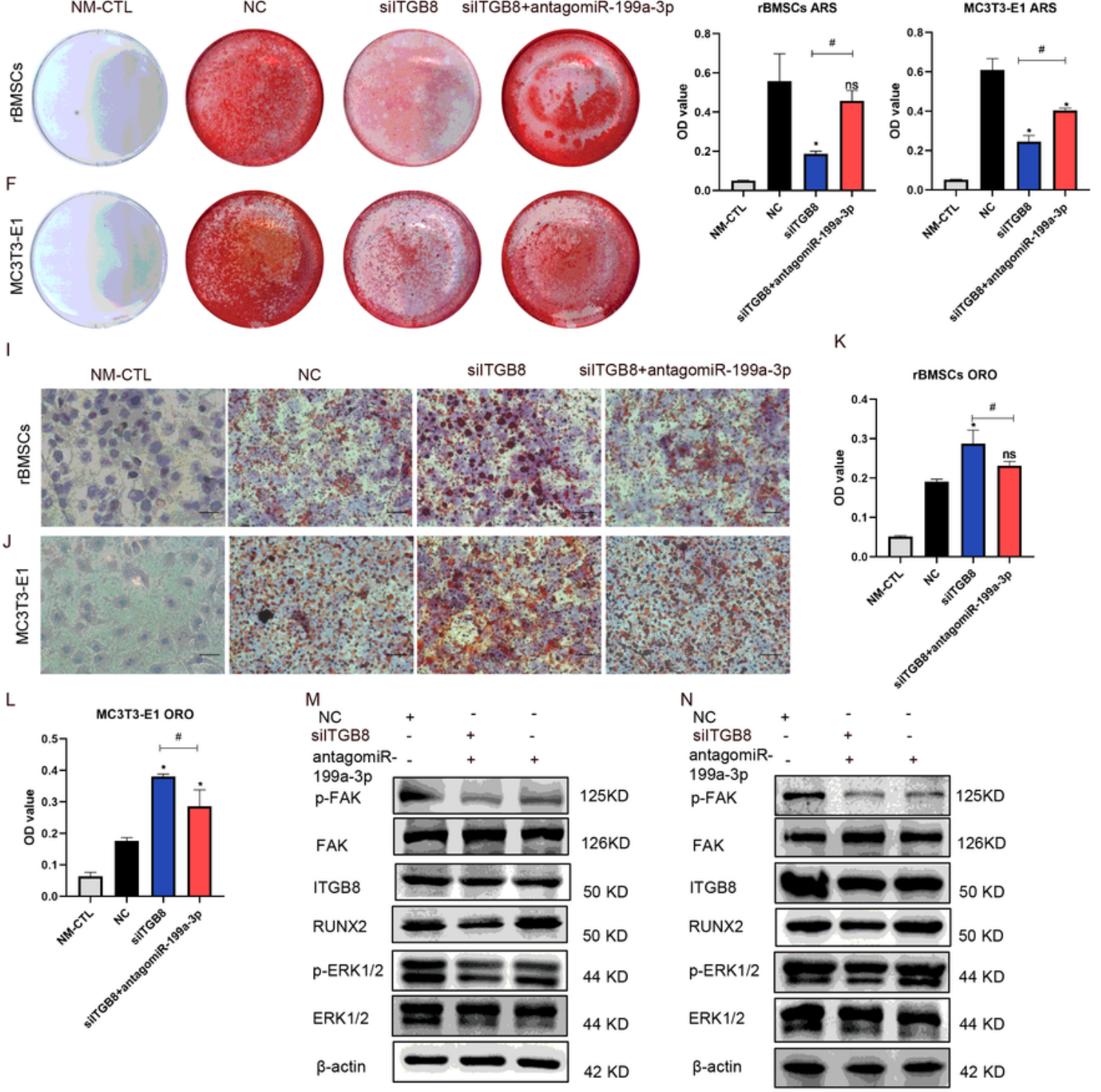

\section{Figure 4}

MiR-199a-3p inhibited osteogenesis of rBMSCs and MC3T3-E1 cells by down-regulating ITGB8 and inactivating the ITGB8-FAK-ERK1/2-RUNX2 axis. A-B WB was used to measure the expression of OCN, ALP, and PPARY in rBMSCs (A) and MC3T3-E1 cells (B). C-D ALP activity of rBMSCs (C) and MC3T3-E1 cells (D). E-F Alizarin red S staining of rBMSCs (E) and MC3T3-E1 cells (F). G-H quantification of Alizarin red S staining. I-J Oil red 0 staining of rBMSCs (I) and MC3T3-E1 cells (J) after cultured in adipocyte 
differentiation medium for 4 weeks (scale bar $=50 \mu \mathrm{m}$ ). K-L quantification of Oil red 0 staining. M-N WB was used to measure the expression of ITGB8, FAK, p-FAK, Erk1/2, p-Erk1/2, and RUNX2 in rBMSCs (M) and MC3T3-E1 cells $(\mathrm{N})$. *P $<0.05$, versus NC group; \#P $<0.05$, versus silTGB8 group. All data were expressed as mean $\pm \mathrm{SD}$.
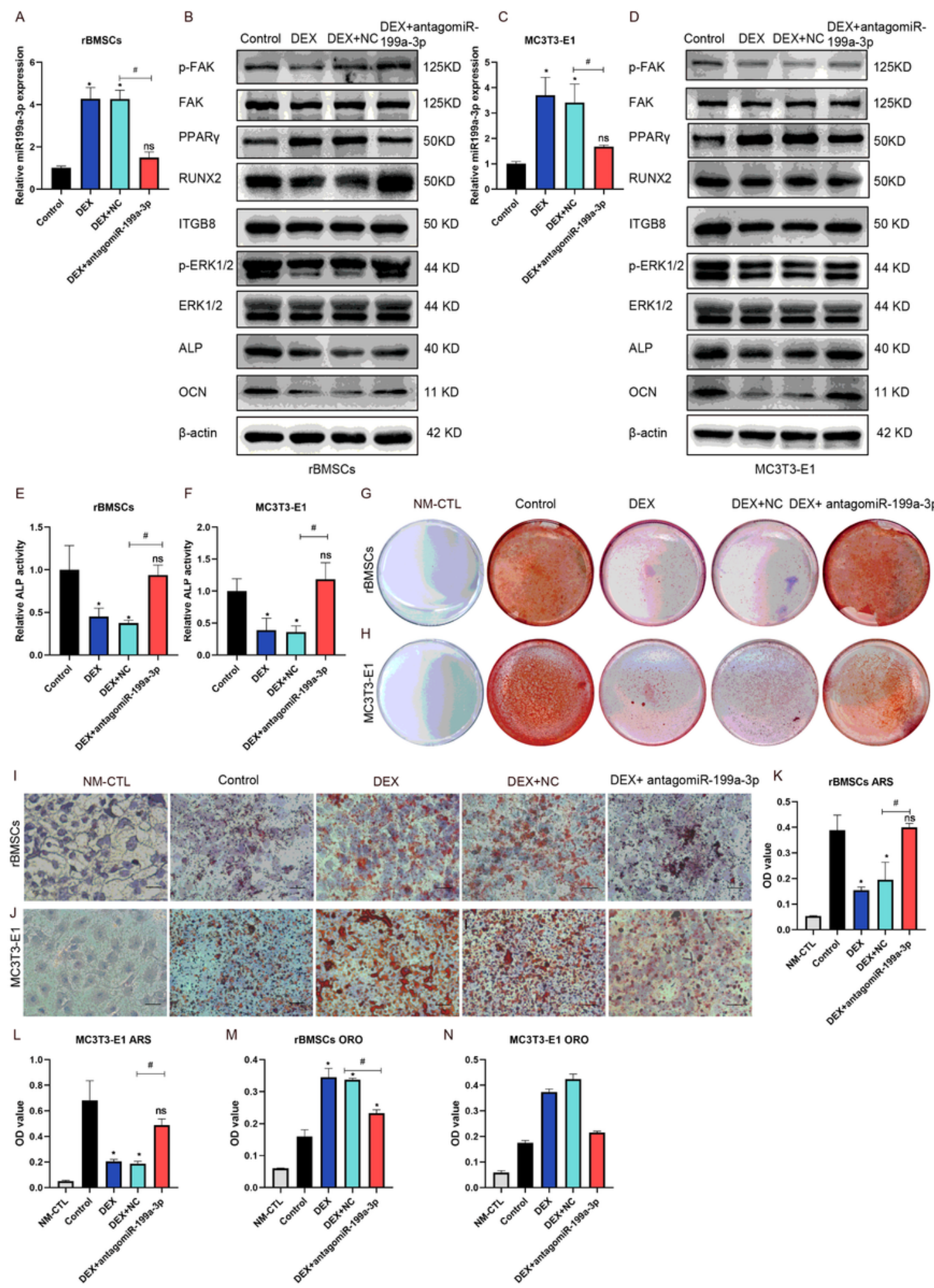

Figure 5 
Silencing of miR-199-3p rescued the suppression of osteogenesis of rBMSCs and MC3T3-E1 cells induced by dexamethasone. A, C RT-PCR was used to measure the expression of miR-199a-3p in rBMSCs (A) and MC3T3-E1 cells (C) in control, DEX, DEX+NC, and DEX+antagomiR-199a-3p group. B, D WB was used to detect the expression of ITGB8, FAK, p-FAK, Erk1/2, p-Erk1/2, OCN, RUNX2, ALP, and PPARY in rBMSCs (B) and MC3T3-E1 cells (D). E-F ALP activity of rBMSCs (E) and MC3T3-E1 cells (F). G-H Alizarin red S staining of rBMSCs $(\mathrm{G})$ and MC3T3-E1 cells $(\mathrm{H})$. I-J Oil red O staining of rBMSCs (I) and MC3T3-E1 cells $(\mathrm{J})($ scale bar $=50 \mu \mathrm{m})$. K-L quantification of Alizarin red $S$ staining. M-N quantification of oil red staining. ${ }^{*} P<0.05$, versus control group; $\# P<0.05$, versus $D E X+N C$ group. $D E X$ : dexamethasone. All data were expressed as mean \pm SD. 


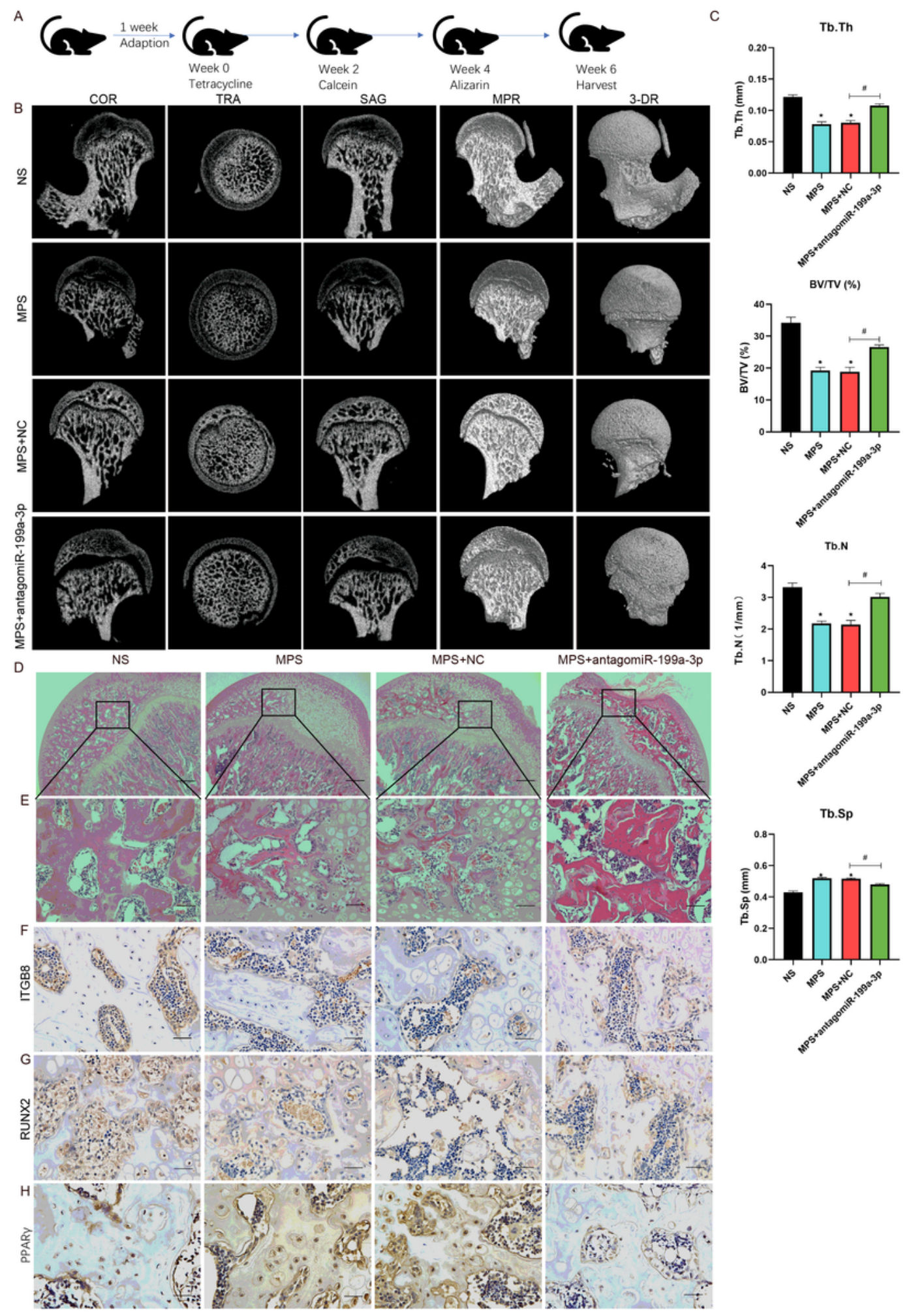

Figure 6

Silencing miR-199a-3p protects GC-induced SANFH damage in vivo. A The diagram of the animal experiments B COR, TRA, SAG, MPR, and 3-DR images of rat femoral heads. COR, coronal; TRA, transverse; SAG, sagittal; MPR, multiplanar reconstruction; 3-DR, three-dimensional reconstruction. C Quantitative analysis of micro-CT scanning. BV/TV, bone volume per tissue volume; Tb. N, trabecular number; Tb. Sp, trabecular separation; Tb. Th, Trabecular thickness. D-E H\&E staining of the femoral 
heads (D: scale bar= $200 \mu \mathrm{m}$; E: scale bar= $100 \mu \mathrm{m})$ ). F-H The expression of ITGB8 (F), RUNX2 (G), and PPARY $(\mathrm{H})$ in the femoral head of rats were measured by $\mathrm{IHC}$ staining (scale bar= $50 \mu \mathrm{m})$. ${ }^{\star} \mathrm{P}<0.05$, versus control group; \#P < 0.05, versus MPS+NC group. All data were expressed as mean \pm SD.

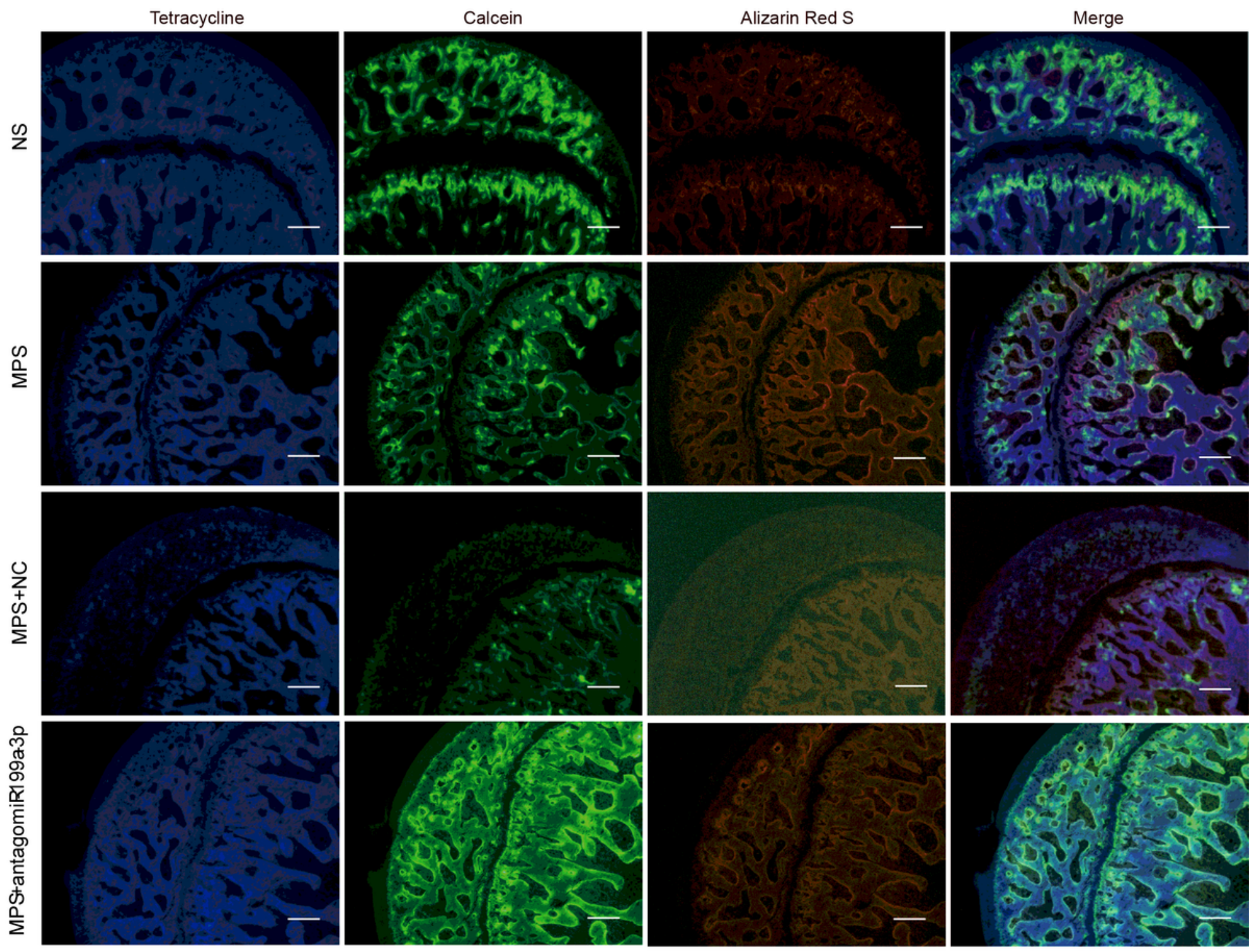

\section{Figure 7}

The protective function of antagomir-199a-3p against SANFH in the rat model. There was significantly decreased new bone formation in the MPS-treated group revealed by the fluorochrome labeling, while the pharmacotherapy with antagomir-199a-3p critically restored bone formation capacity. 